\title{
Superantigen profiles of emm and emm-like typeable and nontypeable pharyngeal streptococcal isolates of South India
}

\author{
Thangarajan Durai Anand ${ }^{1}$, Thangamani Rajesh ${ }^{1,2}$, Jeyaprakash Rajendhran ${ }^{1}$ and Paramasamy Gunasekaran ${ }^{*}$
}

\begin{abstract}
Background: The major virulence factors determining the pathogenicity of streptococcal strains include M protein encoded by emm and emm-like (emmL) genes and superantigens. In this study, the distribution of emm, emmL and superantigen genes was analyzed among the streptococcal strains isolated from the patients of acute pharyngitis.

Methods: The streptococcal strains were isolated from the throat swabs of 1040 patients of acute pharyngitis. The $\mathrm{emm}$ and $\mathrm{emmL}$ genes were PCR amplified from each strain and sequenced to determine the emm types. The dotblot hybridization was performed to confirm the pathogens as true emm nontypeable strains. The presence of eleven currently known superantigens was determined in all the strains by multiplex PCR.

Results: Totally, 124 beta-hemolytic streptococcal strains were isolated and they were classified as group A streptococcus (GAS) [15.3\% (19/124)], group C streptococcus (GCS) [59.7\% (74/124)] and group G streptococcus (GGS) [25.0\% (31/124)]. Among 124 strains, only 35 strains were emm typeable and the remaining 89 strains were emm nontypeable. All GAS isolates were typeable, whereas most of the GCS and GGS strains were nontypeable. These nontypeable strains belong to S. anginosus [75.3\% (67/89)] and S. dysgalactiae subsp. equisimilis [24.7\% (22/89)]. The $\mathrm{emm}$ and $\mathrm{emmL}$ types identified in this study include emm12.0 (28.6\%), stG643.0 (28.6\%), stC46.0 (17.0\%), emm30.11 (8.5\%), emm3.0 (2.9\%), emm48.0 (5.7\%), st3343.0 (2.9\%), emm107.0 (2.9\%) and st5104.2 (2.9\%). Various superantigen profiles were observed in typeable as well as nontypeable strains.

Conclusions: Multiplex PCR analysis revealed the presence of superantigens in all the typeable strains irrespective of their $\mathrm{emm}$ types. However, the presence of superantigen genes in emm and emmL nontypeable strains has not been previously reported. In this study, presence of at least one or a combination of superantigen coding genes was identified in all the emm and emmL nontypeable strains. Thus, the superantigens may inevitably play an important role in the pathogenesis of these nontypeable strains in the absence of the primary virulence factor, $\mathrm{M}$ protein.
\end{abstract}

Keywords: Pharyngeal streptococci, emm typing, superantigen profiling

\section{Background}

Streptococcus pyogenes (group A streptococcus) is a Gram-positive bacterial pathogen. Extracellular surface molecules such as the M protein, hyaluronic acid capsule and fibronectin-binding proteins allow group A streptococcus (GAS) to adhere to, colonise, and invade human skin and mucus membranes under different

\footnotetext{
* Correspondence: gunagenomics@gmail.com

'Department of Genetics, Centre for Excellence in Genomic Sciences, School of Biological Sciences, Madurai Kamaraj University, Madurai-625 021, India Full list of author information is available at the end of the article of the article
}

environmental conditions [1]. GAS is the causative agent of acute bacterial pharyngitis, scarlet fever and impetigo [2] and is responsible for a wide variety of life threatening diseases in humans. The chronic infections lead to acute rheumatic fever (ARF) and rheumatic heart disease (RHD). In recent years, the GAS infections and associated complications are emerging in developing countries like India. More than 60 GAS virulence factors were identified, which were responsible for adherence to epithelial cells, internalization, invasion, systemic toxicity, immune modulation and evasion, cellular or tissue
C Biomed Central 
disruption and bacterial transmission [3]. The major virulence factor reported in GAS is the $M$ protein encoded by emm gene. The $M$ protein confers resistance to phagocytosis by polymorphonuclear leukocytes [4] and binds to complement control factors and thereby prevents the activation of alternate complement pathway [1]. The GAS produces serum opacity factor (SOF) both in extracellular and membrane-bound form [5], which is involved in GAS-host cell adherence process [6].

In 1935, group C streptococcus (GCS) and group G streptococcus (GGS) were first identified as human pathogens [7]. The infections with GCS and GGS can also cause acute pharyngitis, necrotizing fasciitis, sepsis, soft tissue infections and streptococcal toxic shock-like syndrome [8]. S. dysgalactiae subsp. equisimilis and $S$. anginosus are the two important species of GCS/GGS groups. Streptococcus dysgalactiae subsp. equisimilis can cause the whole spectrum of infections caused by $S$. pyogenes $[9,10]$. A high rate of pharyngeal carriage of GCS and GGS in acute rheumatic fever (ARF) patients has also been reported [11].

GAS, GCS and GGS possess common virulence mechanisms including the expression of streptokinase, hyaluronidase, C5a peptidase and M protein [12]. Recently, the presence of GAS virulence genes encoding streptolysin S and glyceraldehyde-3-phosphate dehydrogenase has been reported in bovine $S$. dysgalactiae subsp. dysgalactiae (GCS) and human S. dysgalactiae subsp. equisimilis (GCS/GGS) [13]. Several reports indicated that GCS and GGS also possess emm or emmL genes $[12,14]$. The existence of a considerable level of emm gene sequence homology between GAS and GCS/ GGS groups has been reported [15]. The emmL gene polymorphisms have also been identified in human GGS isolates [6]. Several emm and emmL types of streptococci have been reported in India [16].

GAS produces many pyrogenic exotoxins, called streptococcal pyrogenic exotoxins (SPEs). These toxins act as superantigens (SAgs), which are potent $\mathrm{T}$ cell mitogens. Superantigens simultaneously bind to MHC class II antigens and T cell receptor (TCR) molecules and stimulate $\mathrm{T}$ cells to increase the levels of the cytokines TNF- $\alpha$ and IL- $1 \beta$ and T cell mediators like IL- 2 and IFN- $\gamma$ [17]. These cytokines in high concentration cause fever and shock [18].

The present study describes the prevalence of $\mathrm{emm}$, $e m m L$ and SAg genes in the pharyngeal GAS, GCS and GGS strains isolated from a hospital in Tamil Nadu State, India. An attempt was made to determine the relationship between the superantigen profiles and emm types of pharyngeal streptococci. The strains that possess emm or emmL genes are referred to as typeable strains and the strains lacking emm or emmL genes are referred to as nontypeable strains. The presence of superantigen genes has been previously reported in typeable GAS, GCS and GGS strains. The presence of two GAS superantigens, streptococcal mitogenic exotoxin $\mathrm{Z}$ (smeZ) and streptococcal superantigen $(s s a)$ has been reported in GGS emm types, st245 and stG480 [19]. The presence of GAS superantigen, streptococcal pyrogenic exotoxin $M$ (speM), is detected in GGS emm type, stG10 and GCS emm type, stC1400 [19-21]. However, the presence of superantigen genes in nontypeable strains has not been previously reported. Here, we report the occurrence of different superantigen genes in typeable as well as nontypeable strains of pathogenic streptococci.

\section{Methods}

\section{Strain collection and identification}

Bacterial strains were isolated over a period of one-year from January to December 2009 from Govt. District Headquarters Hospital, Virudhunagar, Tamil Nadu, India. A total of 1040 outpatients of Pediatrics Department between the age of 4 and 12 years with sore throat as their primary complaint were selected. Throat swabs were collected by swabbing the throat in the area of tonsils. The swabs were inoculated immediately on $5 \%$ sheep blood agar and the plates were incubated in 5 to $7 \% \mathrm{CO}_{2}$ at $35^{\circ} \mathrm{C}$ for overnight. The isolates were Lancefield serogrouped using HiStrep latex test kit (Hi-Media Laboratories Pvt. Ltd., Mumbai, India) according to the manufacturer's instructions. Pyrrolidonylarylamidase (PYR) test was performed for the differentiation of S. pyogenes from S. dysgalactiae subsp. equisimilis, S. equi subsp. zooepidemicus and S. anginosus. Acid production from trehalose and sorbitol was used to differentiate $S$. dysgalactiae subsp. equisimilis from S. equi subsp. zooepidemicus. Voges-Proskauer test was done for the differentiation of $S$. dysgalactiae subsp. equisimilis and S. equi subsp. zooepidemicus from S. anginosus $[10,22,23]$.

\section{Bacterial strains and culture conditions}

Beta-hemolytic streptococcal isolates were grown in 5\% sheep blood agar and stored in Todd-Hewitt broth with $20 \%$ glycerol at $-80^{\circ} \mathrm{C}$ until used. Escherichia coli DH5a $\left(\mathrm{F}^{-}\right.$end $\mathrm{A} 1$ gln $\mathrm{V} 44$ thi-1 recA1 relA1 gyrA96 deo $\mathrm{R}$ nup $\mathrm{G}$ Ф80dlacZ $\Delta \mathrm{M} 15 \Delta$ (lacZYA-argF) U169, hsdR17 $\left(\mathrm{rK}^{-} \mathrm{mK}^{+}\right)$, $\lambda$-) was grown in LB broth or agar medium. LB medium supplemented with ampicillin $(100 \mu \mathrm{g} / \mathrm{ml})$ was used to select transformants and maintain plasmids.

\section{Antibiotic susceptibility test}

Streptococcal isolates were screened for susceptibility to penicillin $\mathrm{G}$ (10 units), ampicillin $(10 \mu \mathrm{g})$, amoxicillin (20 $\mu \mathrm{g})$, erythromycin $(15 \mu \mathrm{g})$, azithromycin $(15 \mu \mathrm{g})$, tetracycline $(30 \mu \mathrm{g})$, ceftazidime $(10 \mu \mathrm{g})$ and ceftriaxone $(30 \mu \mathrm{g})$ by Kirby-Bauer disc diffusion method on Mueller-Hinton agar supplemented with $5 \%$ sheep blood. S. pyogenes 
ATCC 12384 was used as a positive control. An overnight grown broth culture of test strain was adjusted to $0.5 \mathrm{Mac}-$ Farland's turbidity standard, and then swabbed evenly on the culture plate. Antibiotic discs were placed on the plate and incubated at $37^{\circ} \mathrm{C}$ under an atmosphere containing 5$7 \% \mathrm{CO}_{2}$ for $18 \mathrm{~h}$. Results were interpreted according to the Clinical Laboratory Standard Institute (CLSI) guidelines [24].

\section{Serum opacity factor (SOF) typing}

Serum opacity factor typing of the streptococcal isolates was done using horse serum according to the method of WHO [25].

\section{DNA extraction and PCR amplification of emm and emmL} genes

Genomic DNA was extracted from all the beta-hemolytic streptococci by using spin column kit following manufacturer's instructions (Hi-Media Laboratories Pvt. Ltd., Mumbai, India). The emm gene was amplified with the "all M" primers [26] and emm2F-emm2R primers [27] (Table 1). The emmL gene was amplified with G1F and G1R primers [6]. All the 124 beta-hemolytic streptococci were subjected to PCR using these three primer pairs. PCR reactions were carried out in an Eppendorf mastercycler (Eppendorf AG, Hamburg, Germany) with 35 cycles, each cycle consisting of initial denaturation at $94^{\circ} \mathrm{C}$ for 5 min, denaturation at $94^{\circ} \mathrm{C}$ for $30 \mathrm{~s}$, annealing at $52^{\circ} \mathrm{C}$ for $30 \mathrm{~s}$, elongation at $72^{\circ} \mathrm{C}$ for $2 \mathrm{~min}$ and a final elongation at $72^{\circ} \mathrm{C}$ for $5 \mathrm{~min}$. PCR products were resolved on $1 \%$ agarose gel, stained with ethidium bromide and analyzed in gel documentation unit (Bio-Rad, USA).

\section{emm and emmL typing}

All the amplicons were individually cloned in a PCR cloning vector, pTZ57RT (MBI-Fermentas, St. Leon-Rot,

Table 1 List of primers used in this study

\begin{tabular}{|c|c|c|}
\hline Primer & Sequence $\left(5^{\prime}-3^{\prime}\right)$ & Reference \\
\hline \multicolumn{3}{|c|}{$\frac{\text { emm/emmL typing }}{\text { primers }}$} \\
\hline emm 1"all M" - F & GGGGGGGGATCCATAAGGAGCATAAAAATGGCT & [26] \\
\hline emm 1"all M"- R & GGGGGGGAATTCAGCTTAGTTTCTTCTTGGG & \\
\hline emm $2-F$ & TATTSGCTTAGAAAATTAA & {$[27]$} \\
\hline emm $2-\mathrm{R}$ & GCAAGTTCTTCAGCTTGTTT & \\
\hline$e m m L-G 1 F$ & AAAAATAAGGAGAAAAAATGG & [5] \\
\hline$e m m L-G 1 R$ & TITTAGTTTCTTCTTTGCG & \\
\hline \multicolumn{3}{|c|}{$\underline{\text { Superantigen primers }}$} \\
\hline spec - F & GGTAAATTITTCAACGACACACACATTAAA & \\
\hline spec - R & TGTTGAGATTCTCCCGAAATAAATAGAT & \\
\hline speG - F & GCTATGGAAGTCAATTAGCTTATGCAGAT & \\
\hline speg - R & TTATGCGAACAGCCTCAGAGG & \\
\hline speJ - F & CAATTAAATTACGCATACGAAATCATACCAGTA & \\
\hline speJ - $R$ & ACGAGTAAATATGTACGGAAGACCAAAAATA & \\
\hline spek - F & TATCGCTTGCTCTATACACTACTGAGAGT & \\
\hline spek - R & CCAAACTGTAGTATTTTCATCCGTATTAAA & {$[21]$} \\
\hline spel - F & GGACGCAAGTTATTATGGATGCTCA & \\
\hline spel - R & TTAAATAAGTCAGCACCTTCCTCTTTCTC & \\
\hline speA1-3,5-F & GGTATITGCTCAACAAGACCCCGAT & \\
\hline speA1-3,5-R & TGTGTTTGAGTCAAGCGTTTCATTATCT & \\
\hline speA1-4-F & CAAGAAGTATTTGCTCAACAAGACCCCA & \\
\hline speA1-4-R & TTAGATGGTCCATTAGTATATAGTTGCTTGTTATC & \\
\hline speH -F & TCTATCTGCACAAGAGGTTTGTGAATGTCCA & \\
\hline speH - R & GCATGCTATTAAAGTCTCCATTGCCAAAA & \\
\hline spel -F & AAGGAAAAATAAATGAAGGTCCGCCAT & \\
\hline spel - $R$ & TCGCTTAAAGTAATACCTCCATATGAATTCTTT & \\
\hline spem - F & GCTTTAAGGAGGAGGAGGTTGATATTTATGCTCTA & \\
\hline speM - R & CAAAGTGACTTACTTTACTCATATCAATCGTTC & \\
\hline smeZ-F & CAATAATTTCTCGTCCTGTGTTTGGAT & \\
\hline smeZ-R & GATAAGGCGTCATTCCACCATAG & \\
\hline$s s a-F$ & AATTATTATCGATTAGTGTITITGCAAGTA & \\
\hline$s s a-R$ & AGCCTGTCTCGTACGGAGAATTATTGAACTC & \\
\hline
\end{tabular}


Germany) and sequenced employing universal M13 and M13R primers at Macrogen, Seoul, South Korea. The $\mathrm{emm}$ and $\mathrm{emmL}$ gene sequences were aligned online with sequences available at the CDC database http:// www.cdc.gov/ncidod/biotech/strep/strepblasts.html to identify the emm types.

\section{Dot-blot analysis}

The absence of emm or emmL genes in the genomes of nontypeable strains were confirmed by dot-blot hybridization using complete $e m m$ and $e m m L$ genes as probes. Genomic DNA (100 ng) of all the nontypeable strains were spotted on nylon membranes (Biodyne ${ }^{\circledR}$ A, Pall, USA) and immobilized by UV-cross linking. The complete emm and $e m m L$ genes were labeled with biotin using NEBlot phototype kit (New England BioLabs, MA, USA) and used as the probe. Hybridization and washing steps were performed as per the manufacturer's instructions. Genomic DNA samples of typeable GAS, GCS and GGS isolates were used as positive controls. Further, the membrane was processed with NEB Phototope ${ }^{\circledR}$-Star detection kit to detect the presence or absence of emm and emmL homologs. The luminescence was visualized using ChemiDoc imaging system (UVP, UK).

\section{Multiplex PCR for superantigens}

Multiplex PCR was performed using Qiagen multiplex PCR kit (Qiagen, Hilden, Germany) to check the presence of streptococcal pyrogenic exotoxin C (speC), speG, speJ, speK, speL, speH, speI, speM, speA, streptococcal mitogenic exotoxin $\mathrm{Z}$ (smeZ) and streptococcal superantigen (ssa) in all the streptococcal isolates with the reported primers [21] (Table 1). PCR reactions were performed in an Eppendorf mastercycler with 35 cycles with each consisting of an initial denaturation at $95^{\circ} \mathrm{C}$ for $15 \mathrm{~min}$, denaturation at $94^{\circ} \mathrm{C}$ for $30 \mathrm{~s}$, annealing at $57^{\circ} \mathrm{C}$ for $90 \mathrm{~s}$, elongation at $72^{\circ} \mathrm{C}$ for $90 \mathrm{~s}$ and final elongation at $72^{\circ} \mathrm{C}$ for $10 \mathrm{~min}$. The reaction mixture consisted of $25 \mu \mathrm{l}$ of $2 \mathrm{X}$ Qiagen multiplex PCR master mix, $2 \mathrm{mM}$ of primer mix (adjusted to $5 \mu \mathrm{l}$ ), $200 \mathrm{ng}$ of template DNA (adjusted to 5 $\mu \mathrm{l})$ and the final volume was adjusted to $50 \mu \mathrm{l}$ using sterile deionized water. PCR products were resolved on a $2 \%$ $(\mathrm{w} / \mathrm{v})$ agarose gel and analyzed as described above.

\section{Nucleotide submissions}

The identified $\mathrm{emm}$ and $\mathrm{emmL}$ gene sequences were submitted to GenBank database under the accession numbers HM125069-HM125081, HM449040-HM449043.

\section{Results}

\section{Characteristics of streptococcal isolates}

In the present study, 124 beta-hemolytic streptococcal strains were isolated and they were classified as group A streptococcus (GAS) [15.3\% (19/124)], group C streptococcus (GCS) [59.7\% (74/124)] and group G streptococcus (GGS) [25.0\% (31/124)]. Within GCS strains, 22 isolates were identified as $S$. dysgalactiae subsp. equisimilis and 52 as S. anginosus. In GGS, 16 isolates were identified as $S$. dysgalactiae subsp. equisimilis and 15 were identified as S. anginosus (Table 2). Antibiotic susceptibility pattern of all 124 streptococcal strains revealed that all of them were sensitive to penicillin $\mathrm{G}$ and all other tested antibiotics.

\section{emm and emmL typing}

Among the 124 strains analyzed, only 35 of the strains were found to be positive for either emm or emmL typing identified by the PCR amplification of emm or

Table $2 \mathrm{emm}$ and emmL typeable and nontypeable pharyngeal streptococci isolated in this study

\begin{tabular}{|c|c|c|c|c|c|c|}
\hline \multirow[t]{3}{*}{ emm or emmL type } & \multicolumn{5}{|c|}{ No. of isolates of: } & \\
\hline & \multicolumn{2}{|c|}{ S. pyogenes } & $\begin{array}{c}\text { S. dysgalactiae } \\
\text { subsp. equisimilis }\end{array}$ & \multicolumn{2}{|c|}{ S. anginosus } & \\
\hline & GAS & GCS & GGS & GCS & GGS & \\
\hline emm12.0 & 10 & & & & & $10(8.1)$ \\
\hline stG643.0 & & & 10 & & & $10(8.1)$ \\
\hline$s t C 46.0$ & & 6 & & & & $6(4.8)$ \\
\hline emm30.11 & 3 & & & & & $3(2.4)$ \\
\hline emm3.0 & 1 & & & & & $1(0.8)$ \\
\hline emm48.0 & 2 & & & & & $2(1.6)$ \\
\hline st3343.0 & 1 & & & & & $1(0.8)$ \\
\hline emm107.0 & 1 & & & & & $1(0.8)$ \\
\hline stS104.2 & 1 & & & & & $1(0.8)$ \\
\hline Nontypeable & 0 & 16 & 6 & 52 & 15 & $89(71.8)$ \\
\hline Subtotal & 19 & 22 & 16 & 52 & 15 & \\
\hline $\begin{array}{l}\text { Total for } \\
\text { species }\end{array}$ & 19 & & 38 & & 67 & 124 \\
\hline
\end{tabular}

GAS, Group A streptococci; GCS, Group C streptococci; GGS, Group G streptococci. 
emmL genes (0.9 to $1.5 \mathrm{~kb})$ as reported earlier [28]. Of these 35 strains, 19 (54.3\%) were positive for emm gene, while $16(45.7 \%)$ were positive for $e m m L$ gene. All the 19 GAS strains were $\mathrm{emm}$ positive and hence these strains were considered $\mathrm{emm}$ typeable. Of the $16 \mathrm{emmL}$ positive strains, 10 strains were of GGS and the other 6 were GCS and these strains could be considered as $e m m L$ typeable. However, the majority of the isolates [89/124 (71.8\%)] were negative for both emm and emmL genes. Further, to examine the presence of emm or emmL homologs in their genomes, the dot-blot hybridization was performed with $\mathrm{emm}$ and $\mathrm{emmL}$ gene specific probes and genomic DNA. However, no detectable signal was obtained suggesting that these strains were truly emm nontypeable. Of these 89 nontypeable strains, $22(24.7 \%)$ were identified as S. dysgalactiae subsp. equisimilis and $67(75.3 \%)$ were S. anginosus. Within the 22 strains of $S$. dysgalactiae subsp. equisimilis, 16 strains were of GCS and 6 strains were of GGS. Among the 67 strains of S. anginosus, 52 were GCS and 15 were of GGS (Table 2).

The emm or emmL typeable strains were subjected to RFLP analysis to ascertain the $\mathrm{emm}$ type. RFLP analysis of these 35 strains revealed 9 distinct patterns (data not shown). All the amplicons regardless of their RFLP pattern were cloned and sequenced. Sequence analysis revealed that RFLP pattern and sequences were similar, thus representing $9 \mathrm{emm}$ types among the 35 typeable strains (Table 3 ).

\section{Serum opacity factor (SOF)}

Among the $35 \mathrm{emm}$ and $\mathrm{emmL}$ typeable strains, 5 of the GAS strains that belong to emm48.0, emm107.0 and stS104.2 were SOF-positive and the other 30 strains were SOF-negative. Significantly, all the nontypeable GCS and GGS strains were SOF-negative.

\section{Superantigen profiling}

The superantigen profiles (combinations of superantigen genes) of the 124 isolates are summarized in Table 3. The superantigen, speC, was detected in all the strains, whereas speJ, speK, speM and ssa were detected in none of the isolates. Among the 19 GAS typeable strains, 10 emm12 type strains were positive for speC, speG, speL, speA1-4 and smeZ. Ten GGS typeable strains (stG643.0) were positive for speC, speG, speA1-4 and smeZ. Six GCS typeable strains (stC46.0) were positive for six superantigens (speC, speG, speA, smeZ, speH and speI).

Table 3 Superantigen profiles of emm and emmL typeable and nontypeable strains of pharyngeal streptococci.

\begin{tabular}{|c|c|c|c|}
\hline S.No & Superantigen Profiles & No. of typeable strains & No. of nontypeable strains \\
\hline 1. & speC & 0 & 1 \\
\hline 2. & speC+ smeZ & 0 & 6 \\
\hline 3. & spec + speg & $3(\mathrm{emm} 30.11)$ & 0 \\
\hline 4. & speC+ speA1-3,5 & $1(s t 3343.0)$ & 0 \\
\hline 5. & speC+ speG+ speA1-4 & $2(\mathrm{emm} 48.0)$ & 3 \\
\hline 6. & speC+ speG+ speA1-3,5 & $1(\mathrm{emm} 107.0)$ & 1 \\
\hline 7. & speC+ speG+spel & 0 & 3 \\
\hline 8. & speC+ speG+ smeZ & 0 & 15 \\
\hline 9. & speC+ speA1-4+ speA1-3,5 & 0 & 1 \\
\hline 10. & speC+ speA1-4+ smeZ & 0 & 4 \\
\hline 11. & speC+ speG+ speA1-4+ speA1-3,5 & $1(\mathrm{emm} 3.0)$ & 3 \\
\hline 12. & speC + speG + speA1-4+ smeZ & $10(s t G 643.0)$ & 27 \\
\hline 13. & speC+ speG+ spel + smeZ & 0 & 6 \\
\hline 14. & speC + speG + spel + spel & 0 & 1 \\
\hline 15. & speC+ speG+ speL+ speA1-4+ smeZ & $10(\mathrm{emm} 12.0)$ & 7 \\
\hline 16. & speC + speG+ speA1-4+ speA1-3,5+ smeZ & 0 & 1 \\
\hline 17. & speC+ speG+speA1-4 + speH+smeZ & 0 & 1 \\
\hline 18. & speC+ speG+ speA1-4+ speA1-3,5+ spel & 0 & 1 \\
\hline 19. & speC+ speG+ speH+ smeZ+ spel & 0 & 1 \\
\hline 20. & speC+ speG+ speA1-4+ smeZ+ spel & 0 & 1 \\
\hline 21. & speC+ speA1-4+speH + smeZ+ spel & 0 & 2 \\
\hline 22. & speC+ speG+ speA1-4+ speA1-3,5+ speH+ smeZ & 0 & 1 \\
\hline 23. & speC+ speG+ speA1-4+ speA1-3,5+ speH+ spel & 0 & 1 \\
\hline 24. & speC+ speG+ speL+ speA1-4+ smeZ+ spel & 1 (stS104.2) & 0 \\
\hline 25. & speC + speG+ speA1-4+ speH+ smeZ+ spel & 0 & 1 \\
\hline 26. & speC+ speG+ speA1-4+ speA1-3,5+speH+smeZ+ spel & $6(s t C 46.0)$ & 1 \\
\hline
\end{tabular}


Interestingly, all nontypeable strains were also found to have at least one superantigen gene (Table 3). Among nontypeable strains, 27 were positive for speC, speG, speA1-4 and smeZ and 15 strains were positive for speC, spe $G$ and smeZ. Maximum of six superantigen genes (speC, speG, speA, smeZ, speH and speI) were detected in a single GCS nontypeable strain ( $S$. anginosus). These results revealed that all the typeable and nontypeable pathogenic beta-hemolytic streptococcal isolates possess at least one superantigen gene.

\section{Discussion}

In the present study, a total of 124 strains were isolated from acute pharyngitis patients. Interestingly, $71.8 \%$ of them were $\mathrm{emm}$ and $\mathrm{emmL}$ nontypeable. The $\mathrm{emm} 12$ was the predominant emm type (28.6\%) among the typeable GAS strains. The prevalence of emm12 was previously reported among the pharyngeal isolates of GAS from Chennai, South India [29]. The presence of emm1, emm3, emm28, emm12, emm4 and emm11 has been reported among the North Indian GAS isolates [30].

In this study, stG643.0 was the predominant emm type (28.6\%) among GGS strains. In contrast, two other GGS emm types, stG485 and stGLP1, were previously reported to be prevalent among 49 GGS isolates from Chennai, India [31]. Other emm types previously reported from India [8] which also represented in this study were emm3 (2.9\%) and stC46 (17.0\%). In addition, the newly identified emm types include emm48.0 (5.7\%), st3343.0 (2.9\%), emm107.0 (2.9\%), stS104.2 (2.9\%), and emm30.11 (8.5\%). According to $\mathrm{CDC}$, these $\mathrm{emm}$ types have already been reported in United States, Mexico, Malaysia and New Zealand, but not from India. Therefore, further studies on the epidemiology of these emm types in India are needed.

The emergence of drug resistance among streptococci to macrolides (erythromycin and clarithromycin) and tetracycline are widely reported in recent years [32-34]. However, in the present study, all the streptococcal strains were susceptible to all the tested antibiotics including $\beta$-lactams, macrolides and tetracycline. Therefore, drug resistance is not a major threat among the streptococci isolated in this study population. However, untreated chronic infections may lead to severe complications such as acute rheumatic fever (ARF) and rheumatic heart disease (RHD).

SOF acts as an adhesin involved in the adherence of GAS to host cells [35]. It has also been reported that the interaction between SOF and the host extracellular matrix protein, fibulin-1, may be involved in the adhesion of GAS to extracellular matrices of the host [36]. Courtney et al. [37] reported that the SOF was expressed at a frequency of $50 \%$ among the clinical isolates of GAS. In contrast, only $14.3 \%$ of typeable GAS isolates were positive for SOF and all GCS and GGS strains were SOF- negative. Similarly, McDonald et al. [15] reported that most of the GCS and GGS strains of $S$. dysgalactiae subsp. equisimilis were SOF-negative. Therefore, SOF may not be the only factor responsible for the adhesion of GAS to the host and some other mechanisms might exist [37]. Some of the GAS adhesins reported other than SOF include lipoteichoic acid, hyaluronic acid capsule, vitronectin-binding protein and collagen-binding protein [2].

We have observed significant differences in the distribution of superantigen genes between typeable and nontypeable strains. The co-occurance of number of superantigen genes was relatively more in typeable than in nontypeable strains. Among 11 superantigens, a maximum of 6 superantigens (speC, speG, speA, speH, speI and $s m e Z$ ) was detected in $17.1 \%$ of typeable strains, whereas this combination was detected in only $1.1 \%$ nontypeable strains. Totally, 26 superantigen profiles have been identified among the typeable and nontypeable strains. Of these, 23 profiles were detected in nontypeable strains and 9 profiles in typeable strains. Only six superantigen profiles were identified in both typeable and nontypeable strains. Other 20 profiles were confined to either typeable or nontypeable strains. As reported previously [38], the most prevalent chromosomally encoded superantingens, speG and smeZ, were detected in $97.1 \%$ and $77.1 \%$ of the typeable strains, respectively. In addition, these two genes were detected in $84.3 \%$ and $83.1 \%$ of nontypeable isolates, respectively. Similarly, Proft et al. [39] have reported the presence of these two superantigens in all the strains of GAS.

A high occurrence of GCS (59.7\%) and GGS (25.0\%) among pharyngeal streptococcal isolates was observed, which is in agreement with previous reports from Spain and Australia [10,15]. It is known that more virulent forms of GCS and GGS could have emerged through the acquisition of superantigen genes from GAS [19]. In this study, many superantigen profiles have been identified among GCS and GGS strains and these strains could be considered as emerging pathogens.

Overall, 67 S. anginosus (52 GCS and 15 GGS) strains and 22 S. dysgalactiae subsp. equisimilis (16 GCS and 6 GGS) strains were nontypeable. Although the presence of superantigens has been reported in typeable GCS and GGS isolates, their presence in nontypeable strains has not been reported so far. However, in our study, compared to the strains of typeable $S$. pyogenes (GAS) and typeable as well as nontypeable $S$. dysgalactiae subsp. equisimilis, more numbers of superantigen profiles have been observed in nontypeable strains of $S$. anginosus (GCS/GGS). In a very recent study [13], the presence of GAS superantigens (speL, speC, speK and speM) has been reported in typeable $S$. dysgalactiae subsp. dysgalactiae (GCS) strains associated with bovine mastitis, but not in 
the human isolates of $S$. dysgalactiae subsp. equisimilis (GCS/GGS). We have identified the presence of superantigens in nontypeable strains of $S$. dysgalactiae subsp. equisimilis (GCS/GGS) associated with acute pharyngitis in humans. Therefore, our findings suggest that, like $S$. pyogenes, S. dysgalactiae subsp. equisimilis and S. anginosus may emerge as potential human pathogens. Some other factors might be involved in the pathogenesis of nontypeable strains in the absence of $M$ protein. The presence of superantigens in nontypeable strains has not been reported so far. The role of superantigens in the pathogenesis of typeable GCS and GGS strains is well documented $[2,19,20]$. However, the role of superantigens in the pathogenic mechanisms of nontypeable strains is yet to be studied.

\section{Conclusions}

A high occurrence of GCS and GGS strains of S. anginosus and $S$. dysgalactiae subsp. equisimilis was observed among the acute pharyngitis patients and most of them were nontypeable. In addition, the presence of superantigens in nontypeable GCS and GGS strains was observed for the first time. Therefore, these strains may emerge as potential human pathogens. Though antibiotic resistance was not observed among these strains, earlier diagnosis and proper antibiotic treatment are required to cure acute pharyngitis and prevent from subsequent complications such as acute rheumatic fever (ARF) and rheumatic heart disease (RHD).

\begin{abstract}
Acknowledgements
Authors thank Dr. N. Aravind Babu, Department of Pediatrics, Govt. District Headquarters Hospital, Virudhunagar, India, for his assistance in the collection of throat swabs. TR thanks UGC, India for the research fellowship under the scheme for meritorious students in Biosciences (No. F. 4-1/2006 (BSR)/5-67/2007). The Centre for Advanced Studies in Functional Genomics, Centre for Excellence in Genomic Sciences and Networking Resource Centre in Biological Sciences are gratefully acknowledged for support facilities.
\end{abstract}

\section{Author details}

'Department of Genetics, Centre for Excellence in Genomic Sciences, School of Biological Sciences, Madurai Kamaraj University, Madurai-625 021, India. ${ }^{2}$ Department of Microbial Engineering, College of Engineering, Konkuk University, 1, Hwayang-dong, Gwangjin-gu, Seoul, 143 701. Korea.

\section{Authors' contributions}

PG designed and coordinated the entire work. TD collected clinical samples and isolated pharyngeal streptococcal strains. TD and TR carried out emm typing. TD and JR carried out superantigen profiling. TD and JR wrote the manuscript and PG corrected the manuscript. All authors have read and approved the final version of the manuscript.

\section{Competing interests}

The authors declare that they have no competing interests.

Received: 20 October 2011 Accepted: 2 February 2012

Published: 2 February 2012

\section{References}

1. Bisno AL, Brito MO, Collins CM: Molecular basis of group A streptococcal virulence. Lancet Infect Dis 2003, 3:191-200.
2. Cunningham M: Pathogenesis of group A streptococcal infections. Clin Microbiol Rev 2000, 13:170-511.

3. Sumby P, Porcella SF, Madrigal AG, Barbian KD, Virtaneva K, Ricklefs SM, Sturdevant DE, Graham MR, Vuopio-Varkila J, Hoe NP, Musser JM: Evolutionary origin and emergence of a highly successful clone of serotype M1 group A streptococcus involved multiple horizontal gene transfer events. J Infect Dis 2005, 192:771-782.

4. Oehmcke S, Podbielski A, Kreikemeyer B: Function of the fibronectinbinding serum opacity factor of Streptococcus pyogenes in adherence to epithelial cells. Infect Immun 2004, 72:4302-4308.

5. Schnitzler N, Podbielski A, Baumgarten G, Mignon M, Kaufhold A: M or Mlike protein gene polymorphisms in human group $\mathrm{G}$ streptococci. J Clin Microbiol 1995, 33:356-363.

6. Timmer AM, Kristian SA, Datta V, Jeng A, Gillen CM, Walker MJ, Beall B, Nizet V: Serum opacity factor promotes group A streptococcal epithelial cell invasion and virulence. Mol Microbiol 2006, 62:15-25.

7. Lancefield RC, Hare R: The serological differentiation of pathogenic and non-pathogenic streptococci from parturient woman. J Exp Med 1935, 61:335-49.

8. Reibmann S, Friedrichs C, Rajkumari R, Itzek A, Fulde M, Rodloff AC, Brahmadathan KN, Chhatwal GS, Patric Nitsche Schmitz D: Contribution of Streptococcus anginosus to infections caused by groups $C$ and $G$ streptococci, Southern India. Emerg Infect Dis 2010, 16:656-663.

9. Nohlgard C, Bjorklind A, Hammar H: Group G streptococcal infections on a dermatological ward. Acta Derm Venereol 1992, 72:128-30.

10. Alberti S, Garcia-Rey C, Garcia-Laorden MI, Dal-Re R, Garcia-de-Lomas J, the Spanish Surveillance Group for Respiratory Pathogens: Survey of emm-like gene sequences from pharyngeal isolates of group $C$ and group $G$ streptococci collected in Spain. J Clin Microbiol 2005, 43:1433-1436.

11. Haidan A, Talay SR, Rohde M, Sriprakash KS, Currie BJ, Chhatwal GS: Pharyngeal carriage of group $C$ and group $G$ streptococci and acute rheumatic fever in an aboriginal population. The Lancet 2000, 356:1167-1169

12. Collins CM, Kimura A, Bisno AL: Group $G$ streptococcal M protein exhibits structural features analogous to those of class I M protein of group A streptococci. Infect Immun 1992, 60:3689-3696.

13. Rato MG, Nerlich A, Bergmann R, Bexiga R, Nunes SF, Vilela CL, SantosSanches I, Chhatwal GS: Virulence gene pool detected in bovine group C Streptococcus dysgalactiae subsp. dysgalactiae isolates by use of a group A S. pyogenes virulence microarray. J Clin Microbiol 2011, 49:2470-9.

14. Smirnov OY, Denesyuk Al, Zakharov MV, Abramov VM, Zavala VP: Protein V, a novel type-II IgG receptor from Streptococcus sp.:sequence, homologies and putative Fc-binding site. Gene 1992, 20:27-132

15. McDonald M, Towers RJ, Andrews RM, Carapetis JR, Currie BJ: Epidemiology of Streptococcus dysgalactiae subsp. equisimilis in tropical communities, Northern Australia. Emerg Infect Dis 2007, 13:1694-1700.

16. Sagar V, Kumar R, Ganguly NK, Chakraborti A: Comparative analysis of emm type pattern of group A streptococcus throat and skin isolates from India and their association with closely related SIC, a streptococcal virulence factor. BMC Microbiol 2008, 8:150

17. Proft T, Moffatt SL, Berkahn CJ, Fraser JD: Identification and characterization of novel superantigens from Streptococcus pyogenes. J Exp Med 1999, 189:89-102.

18. Kotzin BL, Leung DY, Kappler J, Marrack P: Superantigens and their potential role in human disease. Adv Immunol 1993, 54:99-166.

19. Igwe El, Shewmaker PL, Facklam RR, Farley MM, Beneden CV, Beall B: Identification of superantigen genes speM, ssa, and smeZ in invasive strains of beta-hemolytic group C and $\mathrm{G}$ streptococci recovered from humans. FEMS Microbiol Lett 2003, 229:259-264.

20. Kalia A, Bessen DE: Presence of streptococcal pyrogenic exotoxin A and C genes in human isolates of group G streptococci. FEMS Microbiol Lett 2003, 219:291-295

21. Lintges M, Arlt S, Uciechowski P, Plumakers B, Reinert RR, Al-Lahham A, Lutticken $R$, Rink L: A new closed-tube multiplex real-time PCR to detect eleven superantigens of Streptococcus pyogenes identifies a strain without superantigen activity. Int J Med Microbiol 2007, 297:471-478.

22. Fox K, Turner J, Fox A: Role of beta-hemolytic group C streptococci in pharyngitis: Incidence and biochemical characteristics of Streptococcus equisimilis and Streptococcus anginosus in patients and healthy controls. J Clin Microbiol 1993, 31:804-807. 
23. Turner JC, Fox A, Fox K, Addy C, Garrison CZ, Herron B, Brunson C, Betcher G: Role of group C beta-hemolytic streptococci in pharyngitis: Epidemiologic study of clinical features associated with isolation of group C streptococci. J Clin Microbiol 1993, 31:808-811.

24. Clinical and Laboratory Standards institute. Performance standards for antimicrobial susceptibility testing; 15th informational supplement. CLS/ NCCLS document M100- S15 Clinical and Laboratory Standards institute. Wayne, Pa; 2005.

25. Johnson DR: Laboratory diagnosis of group A streptococcal infections Geneva: World Health Organization; 1996.

26. Podbielski A, Melzer B, Lutticken E: Application of the polymerase chain reaction to study the $M$ protein (-like) gene family in beta-hemolytic streptococci. Med Micobiol Immunol 1991, 180:213-227.

27. Centres for Disease Control and Prevention: Protocol for emm-typing. 2009 [http://www.cdc.gov/ncidod/biotech/strep/protocol_emm-type.html]

28. Hookey JV, N A, Saunders NA, Clewley JP, Efstratiou A, George RC: Virulence regulon polymorphism in group A streptococci revealed by long PCR and implications for epidemiological and evolutionary studies. J Med Microbiol 1996, 45:285-293.

29. Menon T, Whatmore AM, Srivani S, Kumar MP, Anbumani N, Rajaji N: emm types of Streptococcus pyogenes in Chennai. Indian J Med Microbiol 2001, 19:161-2.

30. Dhanda V, Kumar R, Thakur JS, Chakraborti A: emm type distribution pattern of group A streptococcus in North India: Need for a new preventive approach. Indian J Med Res 2010, 132:741-744.

31. Menon T, Lloyd C, Malathy B, Sakota V, Jackson D, Beall B: emm type diversity of $\beta$-haemolytic streptococci recovered in Chennai. Indian J Med Microbiol 2008, 57:540-542.

32. Sunaoshi K, Murayama SY, Adachi K, Yagoshi M, Okuzumi K, Chiba N, Morozumi M, Ubukata K: Molecular emm genotyping and antibiotic susceptibility of Streptococcus dysgalactiae subsp. equisimilis isolated from invasive and non-invasive infections. J Med Microbiol 2010, 59:82-88.

33. Bahnan W, Hashwa F, Araj G, Tokajian : emm typing, antibiotic resistance and PFGE analysis of Streptococcus pyogenes in Lebanon. J Med Microbiol 2011, 60:98-101.

34. Imöhl M, Reinert RR, Ocklenburg C, Der Linden MV: Epidemiology of invasive Streptococcus pyogenes in Germany during 2003-2007. FEMS Immunol Med Microbiol 2010, 58:389-96.

35. Gillen CM, H S, Courtney HS, Schulze K, Rohde M, Wilson MR, Timmer AM, Guzman CA, Nizet V, Chhatwal GS, Walker MJ: Opacity factor activity and epithelial cell binding by the serum opacity factor protein of Streptococcus pyogenes are functionally discrete. J Biol Chem 2008, 283:6359-6366.

36. Courtney HS, Li Y, Twal WO, Argraves WS: Serum opacity factor is a streptococcal receptor for the extracellular matrix protein fibulin-1. J Biol Chem 2009, 284:12966-12971.

37. Courtney HS, Hasty DL, Li Y, Chiang HC, Thacker JL, Dale JB: Serum opacity factor is a major fibronectin-binding protein and a virulence determinant of M type 2 Streptococcus pyogenes. Mol Microbiol 1999, 32:89-98.

38. Commons R, Rogers S, Gooding T, Danchin M, Carapetis J, RobinsBrowne R, Curtis N: Superantigen genes in group A streptococcal isolates and their relationship with emm types. J Med Microbiol 2008, 57:1238-1246.

39. Proft T, Webb PD, Handley V, Fraser JD: Two novel superantigens found in both group A and group C streptococcus. Infect Immun 2003, 71:1361-1369.

doi:10.1186/1476-0711-11-3

Cite this article as: Anand et al:: Superantigen profiles of emm and emm-like typeable and nontypeable pharyngeal streptococcal isolates of South India. Annals of Clinical Microbiology and Antimicrobials 2012 11:3.

\section{Submit your next manuscript to BioMed Central and take full advantage of:}

- Convenient online submission

- Thorough peer review

- No space constraints or color figure charges

- Immediate publication on acceptance

- Inclusion in PubMed, CAS, Scopus and Google Scholar

- Research which is freely available for redistribution

Submit your manuscript at www.biomedcentral.com/submit
Biomed Central 